\title{
Investigation of Stimulated Brillouin Scattering Effects in Radio- over-Fiber Distribution Systems
}

\author{
Ling $\mathrm{Hu}$, Aleksandra Kaszubowska and Liam Barry \\ Research Institute for Networks and Communications Engineering, \\ School of Electronic Engineering, \\ Dublin City University, Dublin 9, \\ IRELAND \\ Email: linghu@eeng.dcu.ie
}

\begin{abstract}
Stimulated Brillouin Scattering (SBS) imposes a limitation on the transmitted power in radioover-fiber (RoF) distribution systems. By employing an optical filter at the transmitter that converts the optical microwave signal to Single Side Band (SSB) format, in order to overcome dispersive fading, it is also possible to reduce the effect of SBS on system performance. In this paper the authors compare the cases of filtering the optical microwave signal at the transmitter or the remote site of a radio-over-fibre system, in terms of the effect of SBS on system performance. The authors also investigate and demonstrate how the modulation depth of the optical-microwave data signal influences the Brillouin threshold in RoF distribution systems.
\end{abstract}

Keywords: Optical Communications, Radio-over-fiber (RoF), Stimulated Brillouin Scattering (SBS), Fibre Bragg Grating (FBG), modulation depth.

\section{Introduction}

Radio-over-fiber (RoF) is a technology used to distribute RF signals over analog optical links. In such RoF systems, broadband microwave data signals are modulated onto an optical carrier at a central location, and then transported to remote sites using optical fiber. The base-stations then transmit the RF signals over small areas using microwave antennas [1] [2]. Such a technology is expected to play an important role in present and future wireless networks since it provides an end user with a truly broadband access to the network while guaranteeing the increasing requirement for mobility. In addition, since it enables the generation of millimetrewave signals with excellent properties, and makes effective use of the broad bandwidth and low transmission loss characteristics of optical fibers, it is a very attractively cost-effective and flexible system configuration.

The basic RoF system is a bi-directional analog fiber optic link and consists of a laser/photodiode pair at each site of the link, as well as transmitting and receiving antennas at the remote sites. Signals are generated at a central control station and then distributed to remote sites using single-mode optical fibers. The control station, which can feed many remote sites, is responsible for optical-electrical (o/e) and electrical-optical (e/o) conversions, as well as up-conversion, down-conversion, and processing of the electrical data signals. At the remote sites, only o/e and e/o converters are employed; the o/e converters convert optical signals received from the control station to RF signals and then forward them using the antennas to the end users; the e/o converters convert the RF signals from the antennas to 
optical signals that are then transmitted to the control station [3]. Depending on the system architecture, some type of optical filtering may normally be employed in the such systems either to convert a Double Side Band (DSB) signal to Single Side Band format (to overcome dispersive fading), or to filter out one optical data channel if wavelength division multiplexing (WDM) technology is employed in the system. Indeed, it is possible to use one Bragg filter at the remote site to carry out both these functions simultaneously [1].

Several nonlinear optical effects normally limit the optical power level that can be employed in RoF distribution systems. These effects include Stimulated Brillouin and Raman Scattering (SBS and SRS), Self-Phase Modulation (SPM), and cross-phase modulation and Four-Wave Mixing, for two or more channels [4]. In this work we focus on Stimulated Brillouin Scattering (SBS) since it is likely to have the lowest threshold in systems employing lasers with narrow linewidths. SBS occurs when the incident pump wave generates acoustic waves through the process of electrostriction and the sound waves cause vibrations in the glass lattice that makes up the fiber core [5]. Once the Brillouin threshold is reached, SBS occurs in the fiber, reflecting parts of the input powers launched into the optical fibre and the power is converted into the backward-travelling light, which is named the Stokes wave [6]. The SBS process induces optical power fluctuations which degrade the $\mathrm{Q}$ factor and consequently the bit error rate of an optical communication system. As a result, SBS limits the performance of optical transmission systems and the fiber-input power should normally be kept below the Brillouin threshold [7].

In a single-mode optical fiber the only relevant directions are the forward and the backward directions, and when the scattering angle $\theta=\pi$, the shift reaches the maximum value. Therefore, the optical fiber only allows observation of the Stokes wave in the backward direction. The frequency difference between the incident wave and the Stokes wave is given by $v_{B}$ :

$v_{B}=\frac{2 n V_{A}}{\lambda_{p}}$

where $\lambda_{p}, n$, and $V_{A}$ are the wavelength of the incident pump, the refractive index of the core, and the sound velocity inside the material, respectively [8][9]. If we use the values appropriate for silica fibers, such as $V_{A}=5.96 \mathrm{~km} / \mathrm{s}$ and $\mathrm{n}=1.45, v_{B}=11.1 \mathrm{GHz}$ is obtained at $\lambda_{p}=1.55 \mu \mathrm{m}$. The criterion for the Brillouin threshold is arbitrarily defined as the input optical pump power $\left(\mathrm{P}_{\text {fiber }}\right)$ at which the backward Stokes power $\left(\mathrm{P}_{\text {Stokes }}\right)$ is equal to $\mathrm{P}_{\text {fiber }}$ at the fiber input [4]. If the pump laser is assumed to have a finite linewidth of $\Delta v_{P}$, and the Stokes wave linewidth is given by $\Delta v_{B}$, then the Brillouin threshold for Continuous Wave $(\mathrm{CW})$ light is given by [7]:

$p_{t h r}^{C W} \approx 21 \frac{A_{\text {eff }} K}{g_{o} L_{\text {eff }}}\left(\frac{\Delta v_{B}+\Delta v_{P}}{\Delta v_{B}}\right)$,

where $L_{\text {eff }}$ is the effective interaction length given by 
$L_{e f f} \equiv\left(1-e^{-\alpha L}\right) / \alpha$,

$A_{\text {eff }}$ is the effective core area of the fiber, $\alpha$ is the fiber loss $(\mathrm{dB} / \mathrm{m}), g_{o}$ is the peak Brillouin gain coefficient $\left(g_{o}=4.6^{*} 10^{-11} \mathrm{~m} / \mathrm{W}\right), L$ is the length of the fiber, and $K$ is the polarization factor $(1 \leq K \leq 2)$, which accounts for polarization scrambling between the pump and the Stoke waves [10].

In the case of narrow linewidth with $\Delta v_{B} \geq \Delta v_{P}$, (2) can be reduced to (for CW light):

$$
p_{t h r}^{C W} \approx 21 \frac{A_{e f f} K}{g_{o} L_{e f f}} .
$$

It has been predicted that SBS could be greatly suppressed by proper frequency or phase modulation [11]. In the case of using phase-shift-keying (PSK) modulation, the Brillouin threshold could be 2.5 times higher than that of CW light [7].

From the formula (4), and taking into account the 2.5 times increase in Brillouin threshold for PSK modulation over CW light, we can calculate the predicted Brillouin threshold curve for standard fiber when PSK modulation is used. The results are plotted in Figure 1. Since the polarization factor $K$ is in the range between 1 and 2, we draw two curves when the $K$ is set at 1 and 2 , respectively.

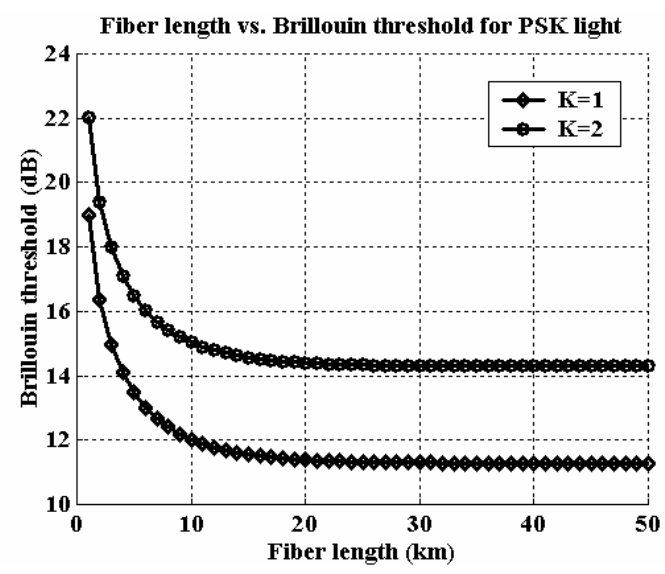

Figure 1. Predicted Brillouin threshold for PSK modulated light (narrow linewidth)

In this paper we examine the effect of SBS in a radio-over-fiber distribution system. We initially demonstrate the difference in system performance that is obtained between placing an optical filter (used for SSB generation, or wavelength channel selection, or both) at the central station or the remote site of the radio-over-fiber distribution system. This difference in system performance is simply due to the insertion loss of the optical filter. We then go on to investigate how the modulation depth of the optical microwave signal will influence the Brillouin threshold in RoF system. This work shows that by using an optical filter which not only generates a SSB signal at the transmitter, but also increases the modulation index of the data signal, the limitations due to SBS can be greatly reduced. 


\section{Experiment setup to measure the Brillouin threshold}

The experimental set up is shown in Figure 2. A $155.520 \mathrm{Mbit} / \mathrm{s}$ NRZ data stream from an Anritsu pattern generator is initially passed through a $117-\mathrm{MHz}$ low-pass filter to minimize the bandwidth of the data signal. Then the signal is mixed with a RF carrier of $18 \mathrm{GHz}$ to generate binary phase-shift keying (BPSK) data signal that is used to directly modulate a single mode laser diode that is biased around $60 \mathrm{~mA}$ and has an operating wavelength of 1550 $\mathrm{nm}$. The optical signal is then amplified using an erbium-doped fiber amplifier (EDFA) before being launched into the optical fiber reel. At the receiver, the detected signal is initially amplified and then downconverted to a baseband signal using a mixer and the $18 \mathrm{GHz}$ local oscillator. At last, the resulting NRZ data signal is amplified before passing through a lowpass-filter and monitored on a $50 \mathrm{GHz}$ oscilloscope.

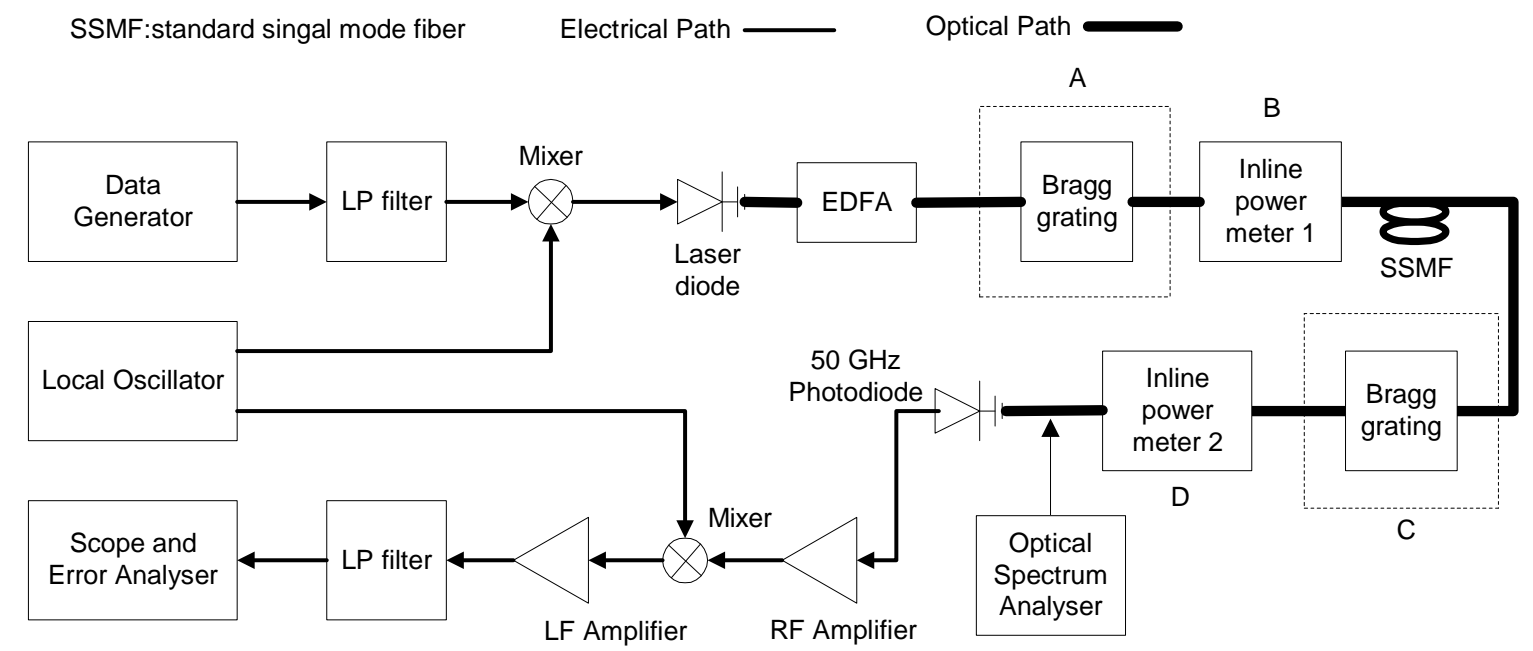

Figure 2. The experiment set up to measure the Brillouin threshold

In this experiment, we use two inline power meters with built-in tunable attenuators (at the positions B and D) to adjust and measure the optical power launched into the fiber reel and the photodiode, respectively. At positions $\mathrm{A}$ and $\mathrm{C}$, we place the Bragg filter according to the experimental demands. The generated optical microwave signal is transmitted over $12.7 \mathrm{~km}$ of standard single mode fiber.

In order to initially measure the Brillouin threshold, we do not need any Bragg filter in the system, which means that there are only fibre patchcords at the position $\mathrm{A}$ and $\mathrm{C}$. In this experiment, we change the optical power launched into the fiber reel by changing the attenuator of inline power meter 1. After the optical signal propagating through the $12.7 \mathrm{~km}$ fiber reel, we adjusted the attenuation of the inline power meter 2 to keep the optical power to be launched into the photodiode constant at $3 \mathrm{dBm}$. It should be noted that with this transmission distance, dispersive fading due to the DSB nature of the signal, does not greatly affect the received signal. From the eye-diagram shown on the oscilloscope the $\mathrm{Q}$ factor of the received signal is calculated according to the definition:

$Q=\frac{v_{H}-v_{L}}{\sigma_{L}+\sigma_{H}}$, 
where $v_{H}$ and $v_{L}$ represent the voltage level of " 1 " signal and " 0 " signal; and $\sigma_{L}$ and $\sigma_{H}$ is the standard deviation of the noise amplitude for " 1 " and " 0 " signals.

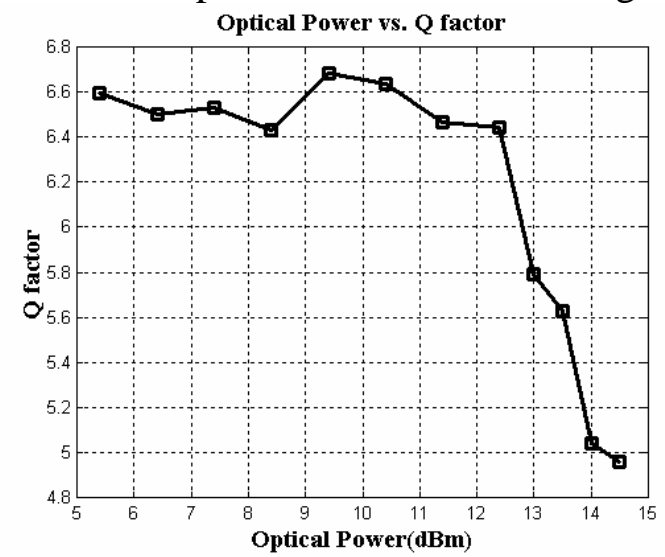

Figure 3. Optical power launched into the fiber reel vs. Q factor

From Figure 3 we see that the Q factor degrades sharply when the optical power launched into the fiber reel reached around $12.5 \mathrm{dBm}$. Since the degradation of $\mathrm{Q}$ factor begins at the Brillouin threshold, the Brillouin threshold for our experimental set-up is about $12.5 \mathrm{dBm}$. This result is within the prediction area shown in Figure 1 for the fiber length $12.7 \mathrm{~km}$.

\section{Overcoming the SBS effect}

In the RoF systems, although the generated optical microwave signals are usually in the DSB format, optical filters may be employed at the receiver (post-filering) to convert the DSB signals to SSB format and eliminate the dispersion fading effects in the fiber. Indeed such a filter could simultaneously demultiplex the desired wavelength channel in RoF WDM systems [1]. Here we propose and demonstrate how the position of the optical filter can influence the performance of a RoF system due to SBS.

\subsection{The System with Post-filtering}

In order to investigate the effect of filter placement on the system performance we carried out the experiments using the above-described methods (i.e. post-and pre- filtering). The first step was to place the Bragg filter at the position " $\mathrm{C}$ " in Figure 2 to measure the effect of the postfiltering. In this experiment, we adjust the pump power of the erbium-doped fiber amplifier (EDFA) to make sure that the optical power launched into the fiber reel reaches $12.5 \mathrm{dBm}$. We connected two fiber reels of $12.7 \mathrm{~km}$ together to obtain a transmission length as $25.4 \mathrm{~km}$. This length makes the SBS effect more severe, and is also the length at which dispersive fading would greatly reduce the received RF power of a DSB signal. The optical power launched into the photodiode is kept at $0 \mathrm{dBm}$ by adjusting the attenuator of inline power meter 2 at the receiver. The received eye-diagram is shown in Figure 4. From this figure it can be seen that the quality of the signal is poor and the eye is very noisy. 


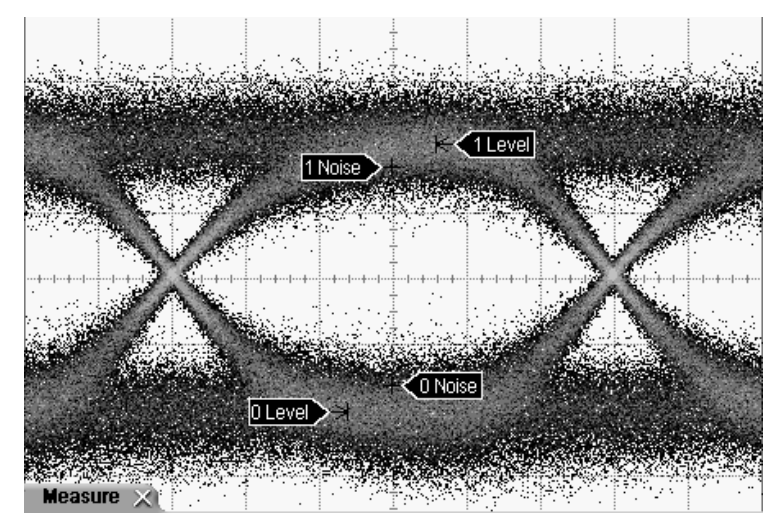

Figure 4. Eye-diagram of received signal with post-filtering

\subsection{The System with Pre-filtering}

In the case of pre-filtering, since the optical filter filters out one side band and has a certain insertion loss, the optical power launched into the fiber is reduced greatly. By placing the insertion loss of the filter at the central station instead of the remote site, the SBS effect could be alleviated. In order to measure the effect of pre-filtering, we move the Bragg filter to the position "A" in Figure 2. Although the optical power before the Bragg filter is still kept at $12.5 \mathrm{dBm}$ (after the EDFA), after passing through the Bragg filter, the inline power meter 1 shows that the optical power launched into the fiber reel fell down to only $7.0 \mathrm{dBm}$. We then adjust the attenuator of the inline power meter 2 to make sure that the optical power launched into the photodiode is kept at $0 \mathrm{dBm}$ (the same for both the pre-filtering and the postfiltering).

In this case, we get a clear and clean eye-diagram, as can be seen in Figure 5. Since the optical power launched into the optical fiber is lower than the Brillouin threshold, we successfully avoid the effect of SBS. The improvement in systems performance between the two cases is essentially the insertion loss of the optical filter employed.

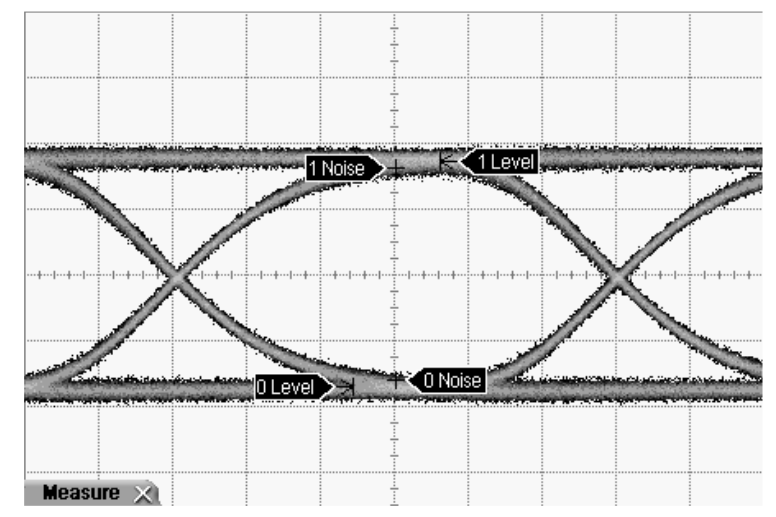

Figure 5. Eye-diagram of received signal with pre-filtering

\section{The influence of the modulation depth on SBS effect}


From formula (4) we know that the linewidth $\Delta v_{P}$ of the laser strongly influence the Brillouin threshold: the wider the $\Delta v_{P}$, the higher the Brillouin threshold. Hence, narrower linewidth signals are more prone to SBS effects. In our RoF system, the linewidth of the optical carrier is much narrower than that of a sideband (which is carrying the data). Therefore, the carrier signal is the main cause of SBS, and reducing the power of the carrier (increasing the modulation depth of the signal) may allow us to reduce the limitation caused by SBS. The modulation depth is expressed in terms of the carrier to sideband ratio (CSR), which is defined as the difference in the optical power between the carrier and a first-order sideband [12]. To vary the modulation index of the generated optical microwave we use the same fixed Bragg filter that was employed earlier, and we use temperature control to vary the emission wavelength of the laser relative to the center of the filter pass band. By doing so we can obtain different values of CSR (i.e. different modulation depths). Figure 6 (a)\&(b) show the spectrum of the signal with the CSR of $1.8 \mathrm{~dB}$ and $23.6 \mathrm{~dB}$ respectively.

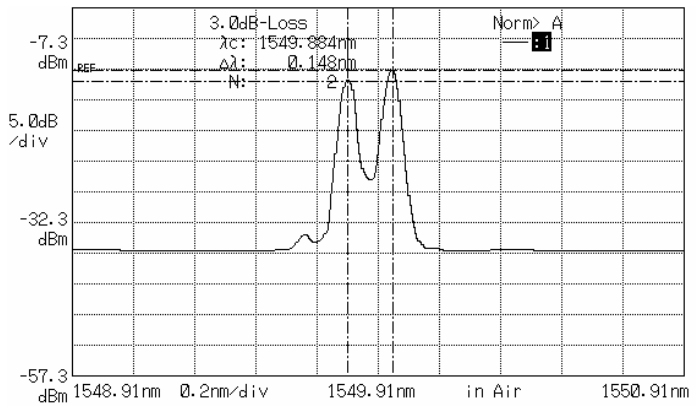

Figure 6 (a). Carrier to Sideband Ratio of $1.8 \mathrm{~dB}$

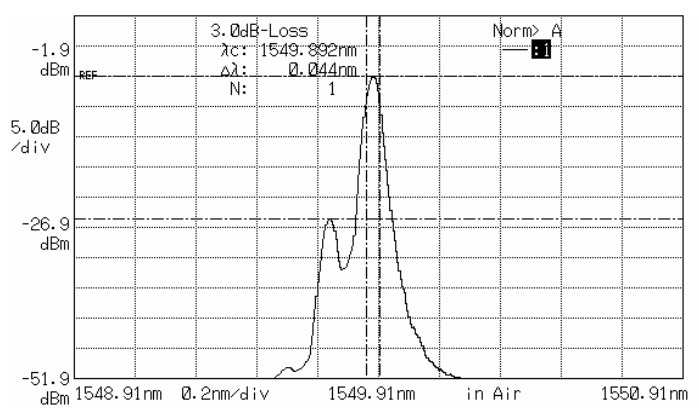

Figure 6 (b). Carrier to Sideband Ratio of $23.6 \mathrm{~dB}$

To investigate the impact of the CSR on the SBS threshold we used an experimental set-up similar to figure 2 . The emission wavelength of the data signal was changed relative to the bandpass profile of the filter to vary the CSR, and a variable attenuator before the transmission fiber was used to keep the launched power constant as the CSR was varied. We choose two different optical launch powers, $6 \mathrm{dBm}$ and $12.5 \mathrm{dBm}$, and in both cases the variable attenuator before the detector is used to keep the received power constant at $0 \mathrm{dBm}$. Figure 7 shows how the Q-factor of the received signal changes when the modulation index is varied for the two launched powers. 


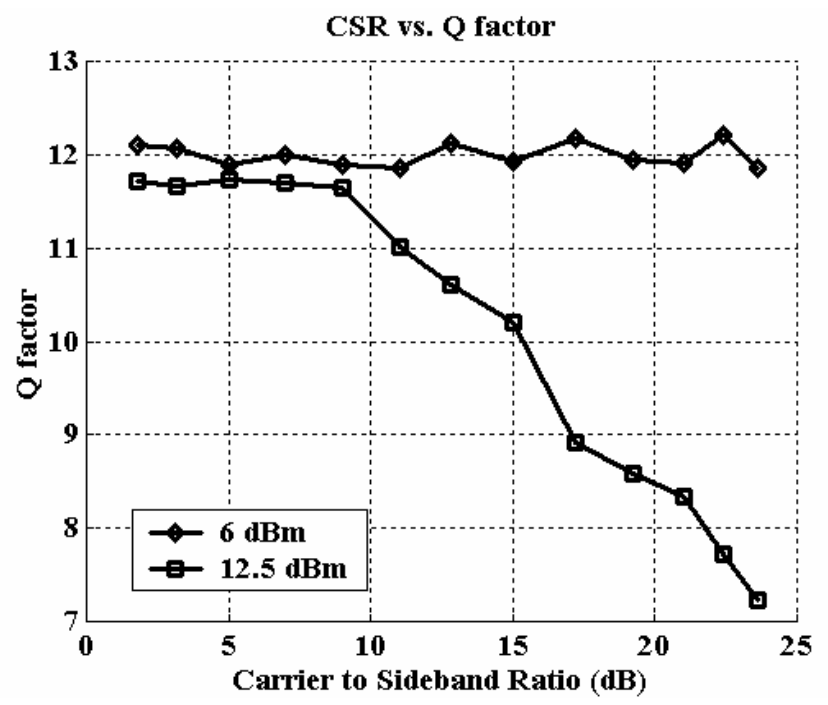

Figure 7. Carrier to Sideband Ratio vs. Q factor

Since $6 \mathrm{dBm}$ is far below the Brillouin threshold, the CSR does not have any influence on the system performance. When the optical power is increased to $12.5 \mathrm{dBm}$, which was the initial Brillouin threshold measured for our system, the situation becomes different. In this case the higher the CSR, the worse the Q factor (the lower the Brillouin threshold). This result shows that the carrier is the main cause of the SBS induced noise in RoF distribution systems. Hence, suppressing the carrier power to change the CSR, reduces the effect of SBS on system performance.

\section{Conclusions}

In this paper, we investigated the effects of SBS on the RoF distribution systems. Many RoF systems may use optical filtering at remote sites to overcome dispersive fading problems, and/or filter out one specific wavelength channel. We have demonstrated that if it feasible to put this filter at the central station of the RoF system, rather that the remote site, then the system performance may be improved due to a reduction in the limitations imposed by SBS. Moreover, we investigated how the modulation depth of the optical microwave signal can affect the SBS effect. The experiment showed that increasing the modulation index increases the Brillouin threshold, as it is primarily the carrier power that induces the SBS effect. Thus, to further reduce the limitations on system performance due to SBS, it may be advantageous to ensure that any optical filter used at the central station not only converts the optical microwave signal to SSB format, but also increase the modulation index of the transmitted signal.

\section{References}

[1] Kaszubowska, A.; Anandarajah, P.; Barry, L.P; Photonics Technology Letters, IEEE, Volume: 16, Issue: 2, Feb. 2004, Pages: 605 - 607.

[2] B.Wilson, Z. Ghassemlooy and I. Darwazeh; Analog Optical Fibre communications, London: IEE Press, 1995. 
[3] Kaszubowska, A.; Anandarajah, P.; Barry, L.P.; Transparent Optical Networks, 2001. Proceedings of 2001 3rd International Conference on, 18-21 June 2001, Pages: 271 - 274.

[4] Govind P. Agrawal, Nonlinear fiber optics, Academic press, 1995.

[5] Fishman, D.A.; Nagel, J.A.; Lightwave Technology, Journal of, Volume: 11, Issue: 11, Nov. 1993, Pages: $1721-1728$

[6] Chraplyvy, A.R. Lightwave Technology, Journal of, Volume: 8, Issue: 10, Oct. 1990, Pages: $1548-1557$.

[7] Aoki, Y.; Tajima, K.; Mito, I.; Lightwave Technology, Journal of, Volume: 6, Issue: 5, May 1988, Pages: $710-719$

[8] Yeniay, A.; Delavaux, J. -M. and; Toulouse, J. Lightwave Technology, Journal of, Volume: 20, Issue: 8, Aug. 2002, Pages: 1425 - 1432.

[9] Shiraki, K.; Ohashi, M.; Tateda, M.; Lightwave Technology, Journal of, Volume: 14, Issue: 1, Jan. 1996, Pages: 50 - 57

[10] Stolen, R.; Quantum Electronics, IEEE Journal of, Volume: 15, Issue: 10, Oct 1979, Pages: $1157-1160$

[11] E.Lilchtman, R.G. Waarts, and A.A. Friesem, Lightwave Technology, Journal of, Volume: 7, Issue: 1, Jan. 1989, Pages: 171 - 174

[12] Attygalle, M.; Lim, C.; Pendock, G.J.; Nirmalathas, A.; Edvell, G.; Photonics Technology Letters, IEEE, Volume: 17, Issue: 1, Jan. 2005, Pages: 190 - 192 